\title{
A Novel Wideband Miniaturized-Element Frequency Selective Surface
}

\author{
Semyoung Oh, ${ }^{1}$ Hanjun Lee, ${ }^{1}$ Joo-Ho Jung, ${ }^{2}$ and Gil-Young Lee ${ }^{1}$ \\ ${ }^{1}$ Electronics and Communication Engineering Department, Air Force Academy, Cheongwon, Chungbuk 335-1, Republic of Korea \\ ${ }^{2}$ Electrical and Computer Engineering Division, Pohang University of Science and Technology, \\ Pohang, Kyungbuk 790-784, Republic of Korea
}

Correspondence should be addressed to Gil-Young Lee; gylee45@gmail.com

Received 22 June 2014; Accepted 2 September 2014; Published 21 September 2014

Academic Editor: Giampiero Lovat

Copyright (c) 2014 Semyoung Oh et al. This is an open access article distributed under the Creative Commons Attribution License, which permits unrestricted use, distribution, and reproduction in any medium, provided the original work is properly cited.

\begin{abstract}
This letter presents a novel wideband miniaturized-element frequency selective surface (MEFSS). The simulation and measurement results show that the bandwidth of the proposed MEFSS is remarkably enhanced compared to that of an original second-order MEFSS while its size and total thickness are still small. A parametric study is also conducted to understand the operating mechanism of the proposed structure. The phenomenon observed in the parametric study is explained with an equivalent circuit model.
\end{abstract}

\section{Introduction}

Frequency selective surfaces (FSSs) have been widely investigated for use as microwave and optical spatial filters in various applications. Similar to microwave circuit filters, FSSs transmit or/and reflect electromagnetic (EM) waves in certain frequency ranges. Four types of frequency responses (lowpass, high-pass, band-stop, and band-pass) can be obtained from the surfaces [1-4], but the band-stop and band-pass responses are used more frequently to achieve a precise and elaborate performance. These two window responses can be realized by etching the metallic screens with resonant geometries and their complementaries. Theoretically, the electrical sizes of the resonant structures should be comparable to half the wavelength. This physical restriction is undesirable for practical FSSs having limited dimensions, because it causes insufficiency of the unit cells (under 400 cells corresponding to $10 \lambda_{0} \times 10 \lambda_{0}$ ) which eventually leads to the deterioration of the desired frequency responses [1]. Various technologies have been studied in order to reduce the electrical sizes to under half the wavelength as in [5-8]. Sarabandi and Behdad, who introduced the concept in [8], are particularly notable for the square metallic patches printed on one side of a dielectric substrate and metallic cross wires printed on the other side of the identical substrate. These constitute a first-order FSS, and the electrical sizes of the elements are much smaller than half the wavelength. Although this first-order miniaturizedelement FSS (MEFSS) can produce stable responses in a small area, it has a small bandwidth, low selectivity, and high insertion loss. Fortunately, these shortcomings are easily addressed by cascading the two identical first-order MEFSS layers that form a second-order MEFSS [9].

Recently, a high-resolution satellite radar system (such as synthetic aperture radar) has been intensively studied. In such an application, a multiorder MEFSS composed of multiple first-order MEFSS layers can be used as a wideband spatial filter because of its small size and simplicity. However, there is a serious increase in the overall thickness, which leads to a bulky structure, as well as limitations in its ability to enhance the bandwidth due to the adjacent resonant frequencies of the MEFSS layers. This letter presents a wideband MEFSS that has two resonances at $8.2 \mathrm{GHz}$ and $13.2 \mathrm{GHz}$. In addition, the $3 \mathrm{~dB}$ bandwidth is $2.8: 1$. Despite the wideband characteristic, the unit cell dimensions $(D \times D)$ are small and the overall thickness is only $0.09 \lambda_{0}^{\prime}$ or $0.14 \lambda_{0}$, where $\lambda_{0}^{\prime}$ and $\lambda_{0}$ are the free-space wavelength at the lower and upper resonant frequencies. Furthermore, the bandwidth can be easily reconfigured by changing the metallic component geometry of the proposed MEFSS. To understand the underlying mechanism, the following sections will present and discuss a parametric 


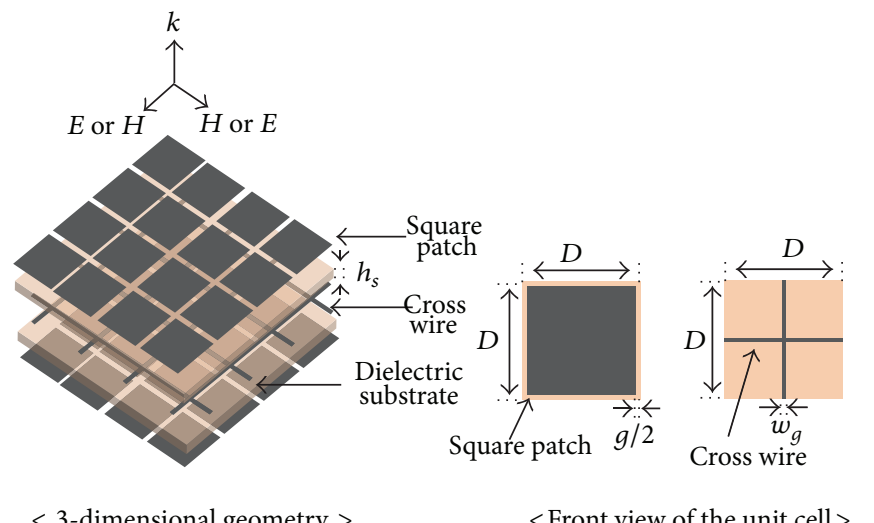

(a)

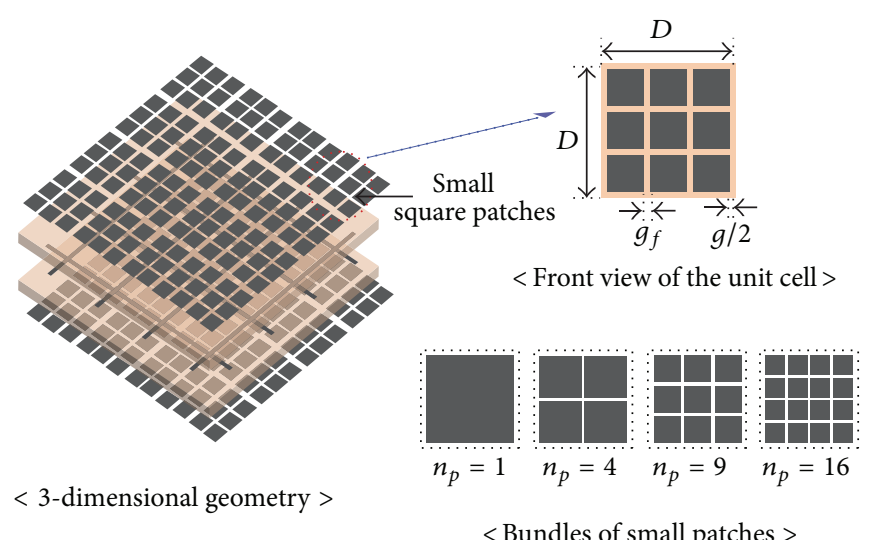

(b)

FIGURE 1: Geometries of the (a) original and (b) proposed MEFSS.

study with an equivalent circuit model. Measurement results will also be provided to validate the simulated results.

\section{Design and Simulation Results}

Figure 1(a) illustrates the three-dimensional geometry of a typical second-order MEFSS in [9]. It consists of two outer metallic layers having square patches with gaps, one inner metallic layer having a cross wire, and two identical dielectric substrates of $\varepsilon_{r}=4.8$ separating the three layers. The structure is simulated with the geometrical parameters in Table 1 using a full-wave EM solver and CST Microwave Studio, and the calculated responses are presented in Figure 2. Here, the parameters are obtained from an optimization process in CST Microwave studio and the following parametric study. As shown in Figure 2, the $3 \mathrm{~dB}$ bandwidth is $2.28: 1$ and the resonant frequencies are $6.63 \mathrm{GHz}$ and $9.8 \mathrm{GHz}$. In order to realize the proposed wideband MEFSS in Figure 1(b), each of the large square patches in the unit cells is replaced with 9 small square patches occupying the dimensions of $(D-g) \times(D-g)$, which are symmetrically arranged and separated with small $0.05 \mathrm{~mm}$ gap of $g_{f}$. Other elements including the dielectric constant $\left(\varepsilon_{r}\right)$ and the thickness $\left(h_{s}\right)$ of the substrates are fixed, and no extra MEFSS layers are added. The full-wave simulation is performed repeatedly with the modified configuration, and the results are plotted over the existing responses in Figure 2. Comparison of the curves in Figure 2 demonstrates a $64 \%$ improvement of the $3 \mathrm{~dB}$ bandwidth and $24 \%$ (lower) and $35 \%$ (higher) movement of the resonant frequencies.

In addition, deviations of stability to obliquely incident angles for transverse electric (TE) and transverse magnetic (TM) polarizations, caused by the structure reconfiguration, are investigated. The transmission coefficients of the proposed design at $0^{\circ}, 15^{\circ}, 30^{\circ}$, and $45^{\circ}$ incident angles for two orthogonal polarizations are obtained through several simulations and are presented in Figure 3. As observed, the proposed wideband MEFSS still works stably at various incident angles for TE and TM polarizations.
TABLE 1: Geometrical parameters of the proposed structure.

\begin{tabular}{lccccc}
\hline Parameters & $h_{s}$ & $D$ & $w_{g}$ & $g$ & $g_{f}$ \\
\hline Values $(\mathrm{mm})$ & 1.6 & 6.5 & 0.75 & 2 & 0.05 \\
\hline
\end{tabular}

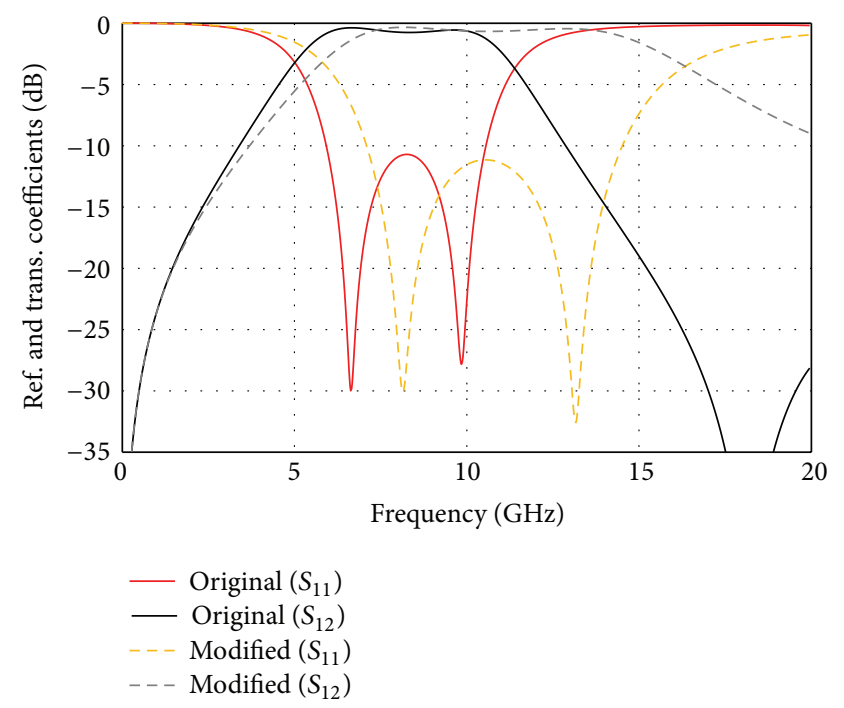

FIGURE 2: Simulated frequency responses of the original and proposed MEFSS at the normal incidence angle.

\section{Parametric Study}

A parametric study is carried out to determine the mechanism underlying the bandwidth enhancement. Six parameters can affect the bandwidth performance: the periodicity of the unit cells $(D)$, the gap width $(g)$, the number of the small patches $\left(n_{p}\right)$, the small gap width $\left(g_{f}\right)$, the wire grid width $\left(w_{g}\right)$, the dielectric constant of the substrates $\left(\varepsilon_{r}\right)$, and the thickness of the substrates $\left(h_{s}\right)$. However, here $n_{p}$ and $g_{f}$ will be investigated, because they are the only parameters representing the geometry of the symmetrically arranged 


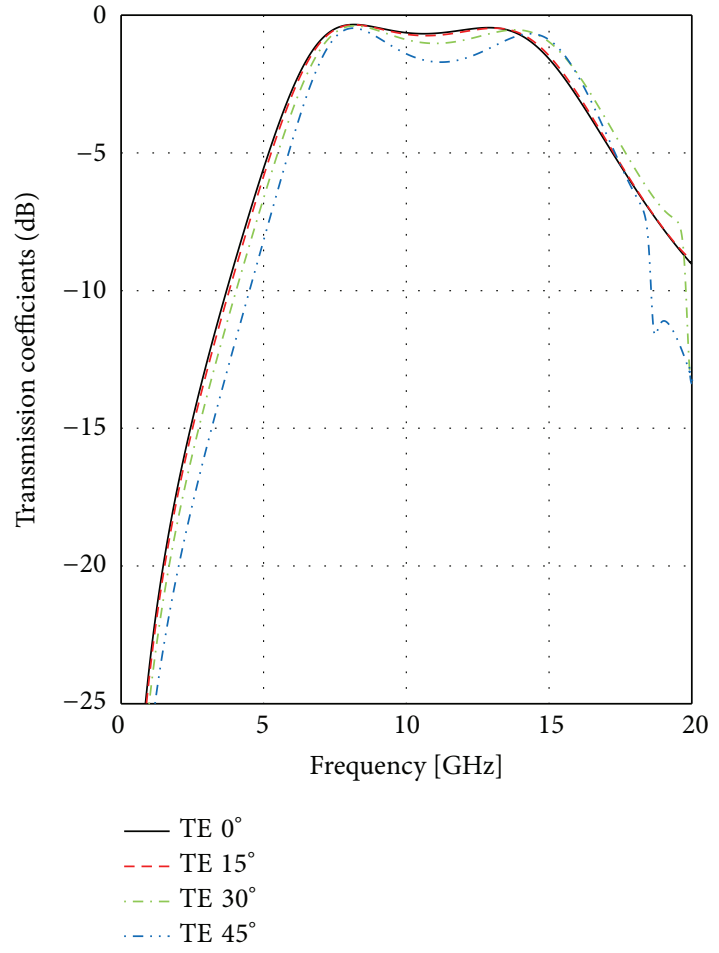

(a)

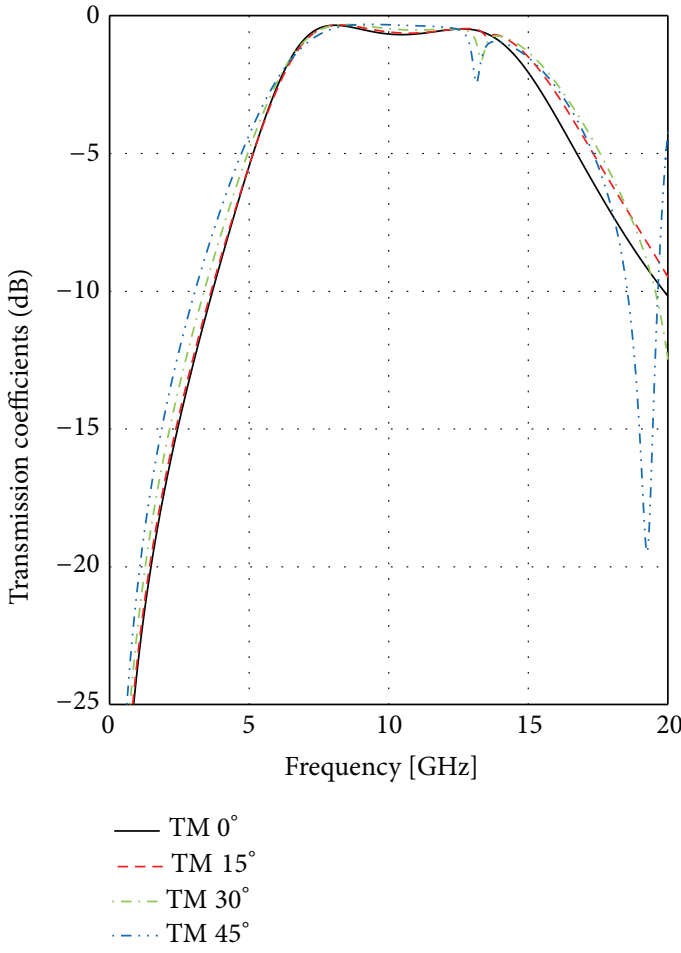

(b)

FIGURE 3: Simulated transmission coefficients at various incident angles for (a) TE and (b) TM polarizations.

small patches with the small gaps. Figure 4 shows the simulated transmission coefficients for the various values of the parameters. As seen in Figure 4, the bandwidth is enhanced when $n_{p}$ and $g_{f}$ are increased. This information indicates that the bandwidth enhancement can be maximized, in principle, by using the largest $n_{p}$ and $g_{f}$. Nevertheless, in this paper they are determined as $9 \mathrm{~mm}$ and $0.05 \mathrm{~mm}$, in order to obtain the responses having the flat pass band with a low insertion loss and a clear roll-off. This also allows the measurement of the structure when the measurement equipment is limited.

The phenomenon examined in the parametric study can be easily explained by using the equivalent circuit model. Figure 5 presents the equivalent circuit of the original secondorder MEFSS, provided in [9]. Here, the capacitors $\left(C_{P}\right)$, the inductor $\left(L_{W}\right)$, and the two short transmission lines with the characteristic impedance of $Z_{C}=Z_{0} / \sqrt{\varepsilon_{r}}$, where $Z_{0}$ is $377 \Omega$, are represented with the outer layers of the patches, the inner layer of the cross wire, and the individual substrates, respectively. All of the circuit elements constitute two coupled parallel LC resonators in the model, and thus the bandwidth of the circuit can be assumed to be inversely proportional to its quality factor ( $Q$-factor) which is proportional to the square root of the value of $C_{P}$ and inversely proportional to the square root of the value of $L_{W}$, as expressed in $Q_{E} \approx$ $\sqrt{C_{P} / L_{W}}$. In summary, the bandwidth of the MEFSS can be expanded by decreasing the value of $C_{P}$ with the fixed value of $L_{W}$.
Interestingly, the value of $C_{P}$ can be estimated directly from the geometrical parameters of the square patch such as $D$ and $g[10]$ :

$$
C_{P}=\varepsilon_{0} \varepsilon_{\mathrm{eff}} \frac{2 D}{\pi} \ln \left(\frac{1}{\sin (\pi g / 2 D)}\right)
$$

where $\varepsilon_{0}$ is the free-space permittivity, $\varepsilon_{\text {eff }}$ is the effective permittivity, $\mu_{0}$ is the free-space permeability, and $\mu_{\text {eff }}$ is the effective permeability. For the proposed wideband MEFSS, each of $D$ and $g$ is replaced with $\left\{D-g-\left(\sqrt{n_{p}}-1\right) \times g_{f}\right\} / \sqrt{n_{p}}$ and $g_{f}$, where $n_{p}$ starts from 4 . Equation (1) is significant to account for the phenomenon observed in Figure 4, in which the smaller values of $C_{P}$ for the wide bandwidth are obtained by increasing $n_{p}$ and $g_{f}$. Additionally, it can also be used to explain frequency shifts occurring with the bandwidth enhancement by using the following equation:

$$
f_{c} \approx \frac{1}{2 \pi \sqrt{C_{P} L_{W}}}
$$

where $f_{C}$ is the center operating frequency of the responses.

The aforementioned speculation can be verified numerically. Table 2 provides the change rates of the $Q$-factor $\left(\Delta Q_{E}\right)$ corresponding to the increase of $n_{p}$, when $g_{f}$ is fixed. These change rates are the ratio of the $Q$-factor of the proposed wideband MEFSS to that of the original structure. They are obtained by utilizing (1) and the relationship with the change 


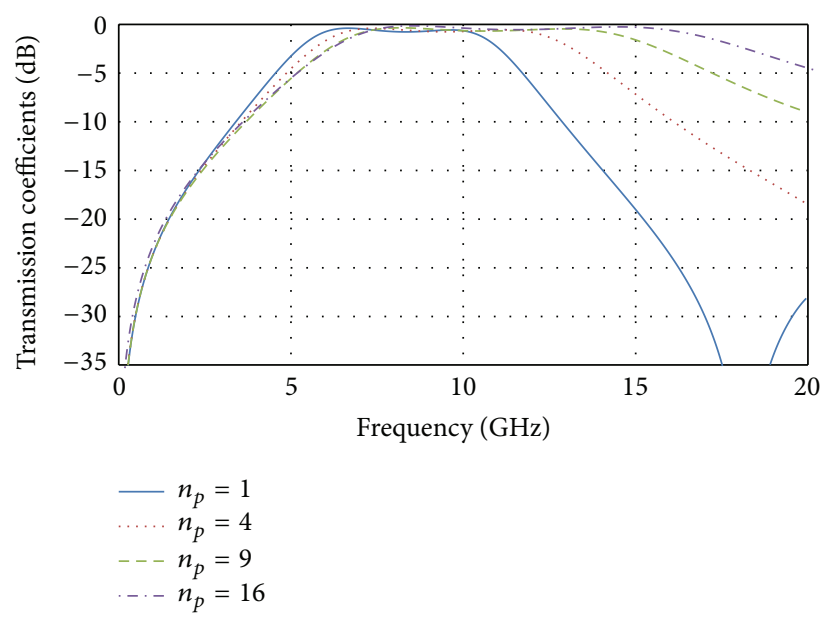

(a)

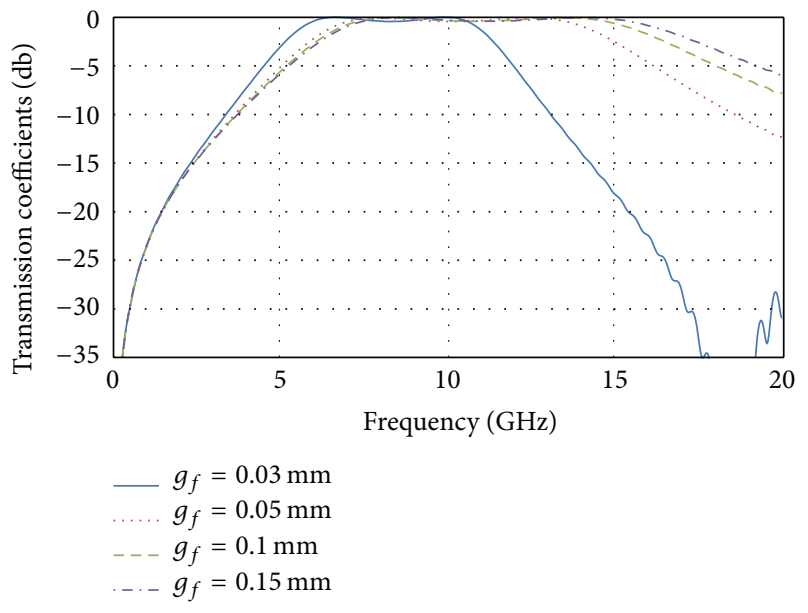

(b)

FIGURE 4: Simulated transmission coefficients of (a) $n_{p}$ and (b) $g_{f}$ at the normal incidence angle.

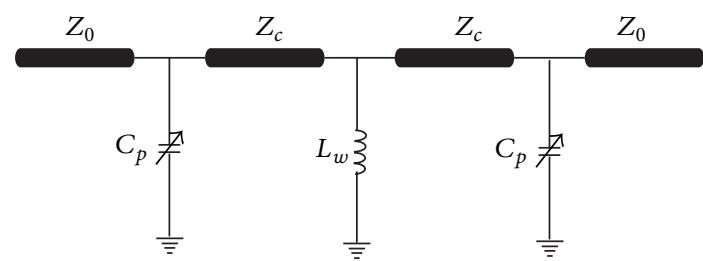

FIGURE 5: Equivalent circuit model, valid for normal incidence, of the original second-order MEFSS [9].

rates of $C_{P}$ in the equivalent model. For convenience, $\varepsilon_{\text {eff }}$ is assumed to be the same as the dielectric constant of the substrates. However, the change rates of the $Q$-factor $\left(\Delta Q_{S}\right)$ can also be calculated directly from the simulated results by using

$$
Q_{S} \approx \frac{f_{c}}{\Delta f}
$$

where $\Delta f$ is the bandwidth of the responses. In Table 2 , these change rates are offered and they are fairly matched with those of the equivalent circuit model, but some discrepancies are also found. This is caused by the values of $C_{P}$ being inaccurate because (1) is perfectly valid without other metallic materials. Another reason is that the coupling effect between the LC resonators is not considered sufficiently.

The change rates of the $Q$-factor with regard to $g_{f}$ can be obtained in similar fashion, but they are not treated in this paper.

\section{Measurement Results}

The proposed wideband MEFSS is fabricated, and it is measured in an anechoic chamber. Pairs of antennas covering from $3 \mathrm{GHz}$ to $18 \mathrm{GHz}$ are placed apart to form a uniform plane wave, and these antennas are connected to an Agilent 5242A network analyzer. The test structure is located at a fixture placed in the middle of the antennas. The measurement
TABLE 2: Change rates of the $Q$-factor of the proposed wideband MEFSS corresponding to the increase in the number of small patches; in the first column, the Q-factor of the original MEFSS is also provided as a reference.

\begin{tabular}{lccccc}
\hline $\begin{array}{l}\text { Number of } \\
\text { the small } \\
\text { patches }\left(n_{p}\right)\end{array}$ & $\begin{array}{c}f_{c} \\
{[\mathrm{GHz}]}\end{array}$ & $\begin{array}{c}\Delta f \\
{[\mathrm{GHz}]}\end{array}$ & $\begin{array}{c}C_{p} \\
{[\mathrm{pF}]}\end{array}$ & $\Delta Q_{E}$ & $\Delta Q_{S}$ \\
\hline- & 8.34 & 6.38 & 0.26 & $Q_{E}$ & $Q_{S}$ \\
& $\left(f_{c}\right)$ & $(\Delta f)$ & $\left(C_{P}\right)$ & & \\
4 & 9.37 & 8.27 & 0.20 & $0.88 Q_{E}$ & $0.86 Q_{S}$ \\
& $\left(1.12 f_{c}\right)$ & $(1.30 \Delta f)$ & $\left(0.79 C_{P}\right)$ & & \\
9 & 10.43 & 10.71 & 0.12 & $0.68 Q_{E}$ & $0.74 Q_{S}$ \\
& $\left(1.25 f_{c}\right)$ & $(1.68 \Delta f)$ & $\left(0.58 C_{P}\right)$ & & \\
16 & 10.90 & 12.97 & 0.08 & $0.56 Q_{E}$ & $0.64 Q_{S}$ \\
\hline
\end{tabular}

is first performed without the prototype. It is then installed to screen the center quadrangle hole of the fixture and the measurement is repeated. The transmission coefficient at normal incidence is obtained from the data processing with the measurement results, as shown in Figure 6. The simulated result is plotted in the same figure for comparison. As Figure 6 shows, good agreement is observed between the two results. However, minor discrepancies are also found. They are caused by the inaccuracy of the fabrication and measurement process.

\section{Conclusion}

This letter proposed a novel wideband MEFSS to support a high-resolution satellite radar system. Even though its size and overall thickness are small, the proposed MEFSS can operate at a wide frequency range. The mechanisms of the bandwidth enhancement are studied with a parametric study and are explained by the equivalent circuit model. The designed MEFSS is fabricated and measured to verify the 


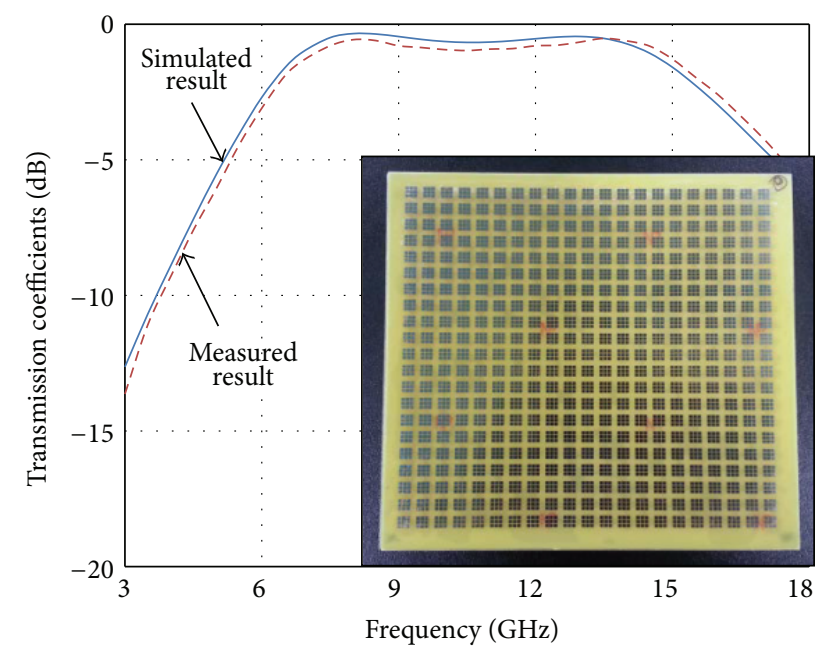

FIGURE 6: Measured and simulated transmission coefficients of the proposed wideband MEFSS. The simulated result is recalculated after considering a bonding film between the dielectric substrates. The bonding film (5318PLA from Coretech Co.) has 6 mil thickness, a dielectric constant of 2.25, and a loss tangent of 0.00031 .

simulation results, and a good agreement is observed between the experiment and simulation results.

\section{Conflict of Interests}

The authors declare that there is no conflict of interests regarding the publication of this paper.

\section{References}

[1] B. A. Munk, Frequency Selective Surfaces: Theory and Design, Wiley-Interscience, New York, NY, USA, 2000.

[2] H. M. Lee and Y. J. Kim, "Double-layered frequency selective surface superstrate using ring slot and dipole-shaped unit cell structure," Journal of Electromagnetic Engineering and Science, vol. 10, no. 3, pp. 86-91, 2012.

[3] G. A. E. Crone, A. W. Rudge, and G. N. Taylor, "Design and performance of airborne radomes : a review," IEE proceedings. Part F. Communications, radar and signal processing, vol. 128, no. 7, pp. 451-464, 1981.

[4] M. Gustafsson, A. Karlsson, A. P. P. Rebelo, and B. Widenberg, "Design of frequency selective windows for improved indoor outdoor communication," IEEE Transactions on Antennas and Propagation, vol. 54, no. 6, pp. 1897-1900, 2006.

[5] G. Yang, T. Zhang, W. Li, and Q. Wu, "A novel stable miniaturized frequency selective surface," IEEE Antennas and Wireless Propagation Letters, vol. 9, pp. 1018-1021, 2010.

[6] H. Liu, K. L. Ford, and R. J. Langley, "Miniaturised bandpass frequency selective surface with lumped components," Electronics Letters, vol. 44, no. 18, pp. 1054-1055, 2008.

[7] W. T. Wang, P. F. Zhang, S. X. Gong, B. L. J. Ling, and T. T. Wan, "Compact angularly stable frequency selective surface using hexagonal fractal configurations," Microwave and Optical Technology Letters, vol. 51, no. 11, pp. 2541-2544, 2009.
[8] K. Sarabandi and N. Behdad, "A frequency selective surface with miniaturized elements," IEEE Transactions on Antennas and Propagation, vol. 55, no. 5, pp. 1239-1245, 2007.

[9] M. Al-Joumayly and N. Behdad, "A new technique for design of low-profile, second-order, bandpass frequency selective surfaces," IEEE Transactions on Antennas and Propagation, vol. 57, no. 2, pp. 452-459, 2009.

[10] N. Marcuvitz, Waveguide Handbook, Boston Technical Publishers, Lexington, Mass, USA, 1964. 

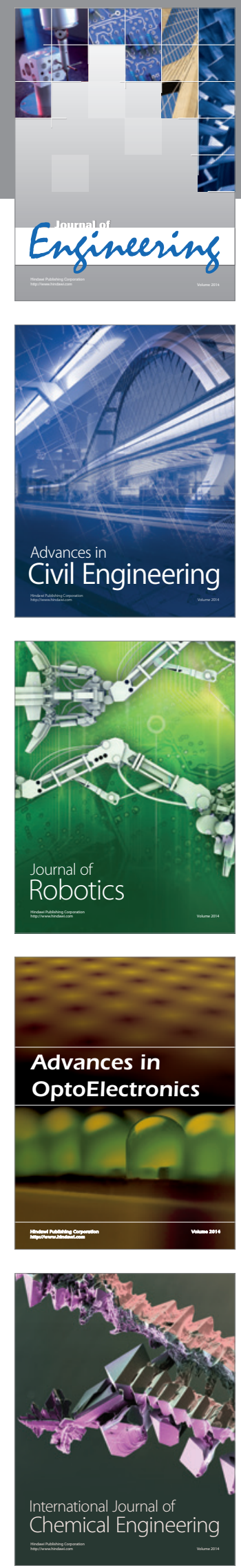

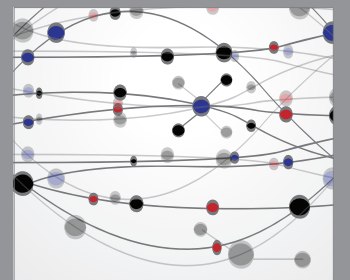

The Scientific World Journal
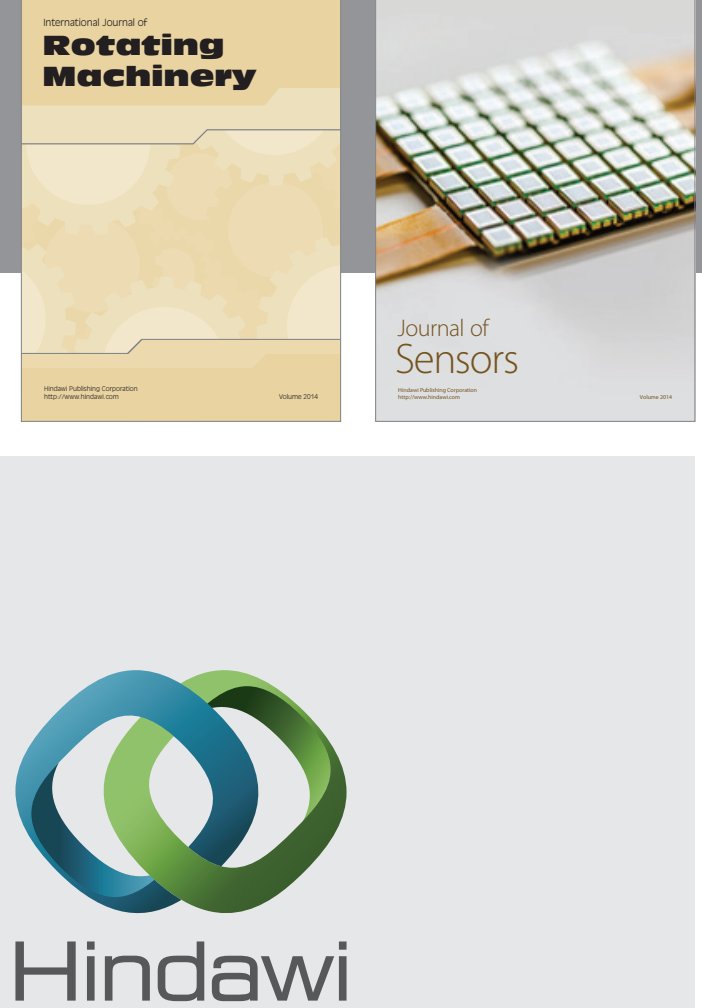

Submit your manuscripts at http://www.hindawi.com
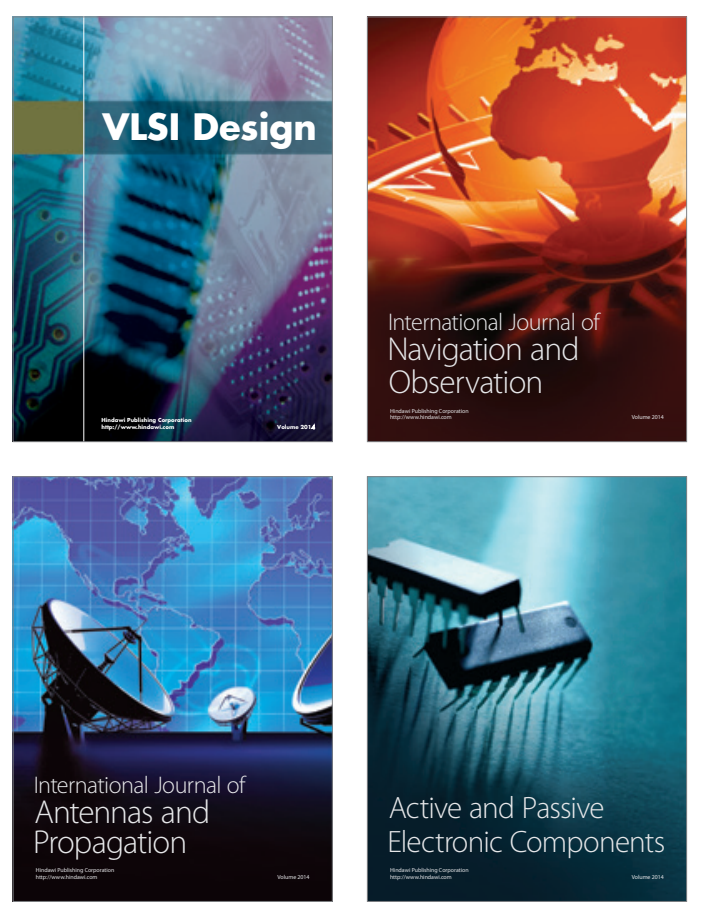
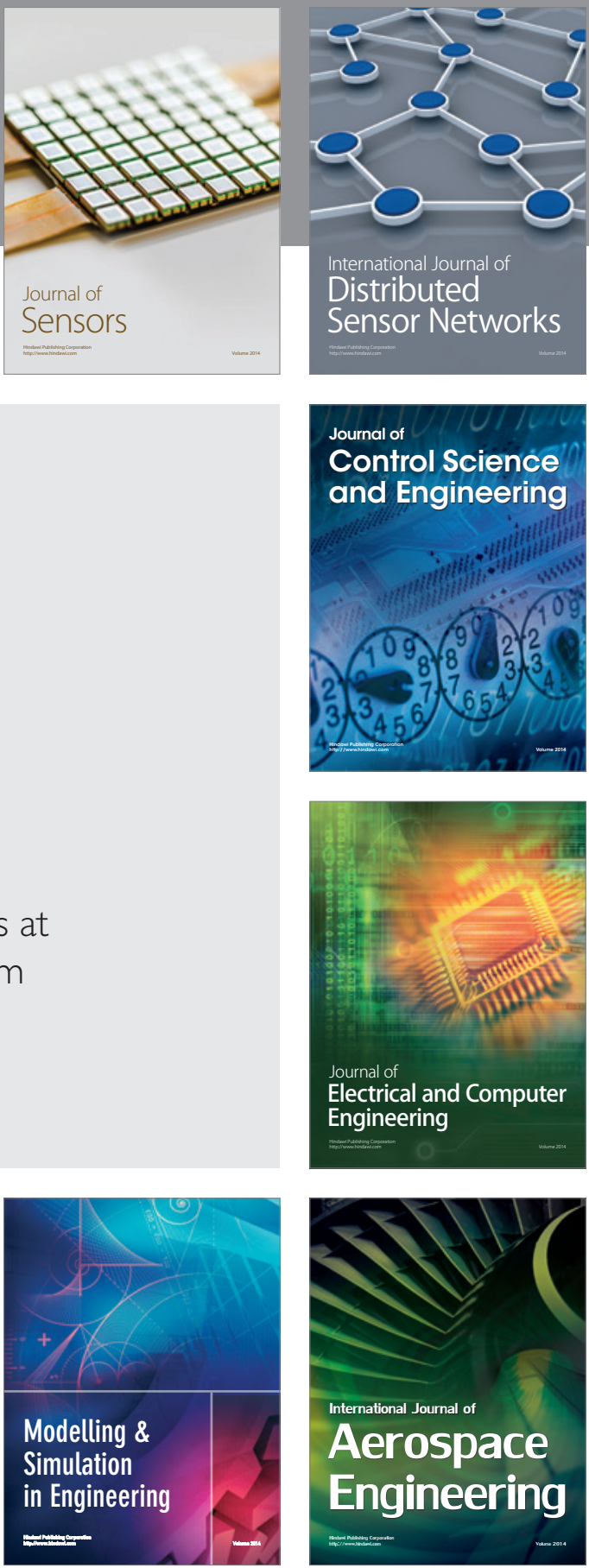

Journal of

Control Science

and Engineering
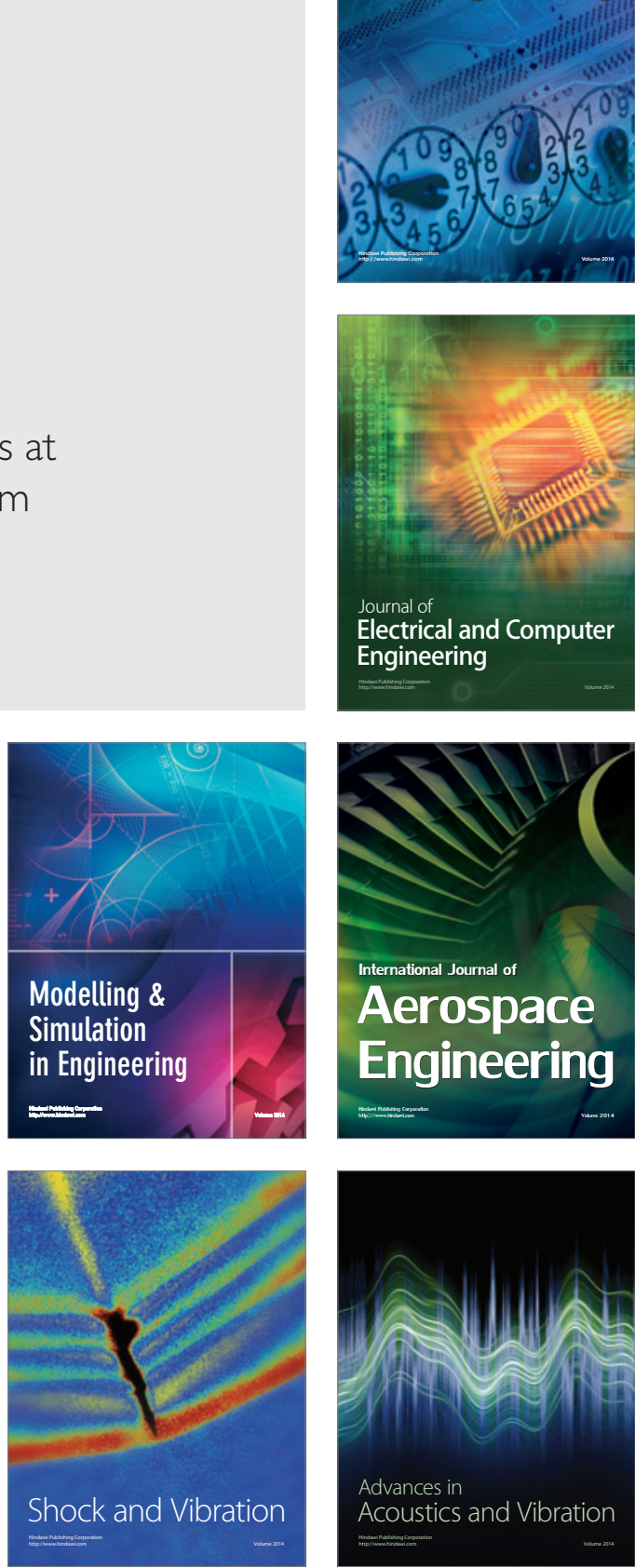